\title{
Load Shared Sequential Routing in MPLS Networks: System and User Optimal Solutions
}

\author{
Gilles Brunet ${ }^{1}$, Fariba Heidari ${ }^{2}$, and Lorne G. Mason ${ }^{2}$ \\ ${ }^{1}$ Videotron Ltd., Montreal, QC, Canada \\ ${ }^{2}$ Department of Electrical and Computer Engineering, \\ McGill University, Montreal, QC, Canada \\ Gilles.Brunet@videotron.com, fariba.heidari@mail.mcgill.ca, \\ lorne.mason@mcgill.ca
}

\begin{abstract}
Recently Gerald Ash has shown through case studies that event dependent routing is attractive in large scale multi-service MPLS networks. In this paper, we consider the application of Load Shared Sequential Routing (LSSR) in MPLS networks where the load sharing factors are updated using reinforcement learning techniques. We present algorithms based on learning automata techniques for optimizing the load sharing factors both from the user equilibrium and system optimum perspectives. To overcome the computationally expensive gradient evaluation associated with the Kuhn-Tucker conditions of the system optimum problem, we derive a computationally efficient method employing shadow prices. The proposed method for calculating the user equilibrium solution represents a computationally efficient alternative to discrete event simulation. Numerical results are presented for the performance comparison of the LSSR model with the user equilibrium and the system optimum load sharing factors in some example network topologies and traffic demands.
\end{abstract}

\section{Introduction}

In the early days of packet switching much attention was given to the routing problem. See [1] for an early survey of routing algorithms where an application of learning techniques to packet routing in data networks was considered. The event dependent routing method described in reference [1] can be applied to either datagram or virtual circuit data networks, however in the case of datagram networks the received packets can be miss ordered. With the appearance of the Internet, destination based IP routing was widely adopted for reasons of scalability and stability in spite of the fact that destination based routing gives the user little control over how his/her traffic is routed. This in turn means that traffic may be routed over congested links (paths) while at the same time alternative less congested paths are available.

The need for better control of traffic routing, also referred to as "traffic engineering", gave rise to the MPLS standard. Multi Protocol Label Switching (MPLS) is a connection oriented framework proposed by the IETF to improve

T. Chahed and B. Tuffin (Eds.): NET-COOP 2007, LNCS 4465, pp. 226-235 2007.

(C) Springer-Verlag Berlin Heidelberg 2007 
traffic engineering, congestion management and QoS provisioning in traditional IP networks [2]. In this framework, constraint-based routing and label swapping replaces the hop-by-hop destination-based routing mechanism used in traditional IP networks. With MPLS, route selection can employ either hop by hop routing or explicit routing. In the explicit routing method, a single Label Switching Router (LSR), usually the ingress LSR, specifies all (or some of) the hops in the Label Switched Path (LSP). Explicit routing gives the designer the ability to control the traffic load distribution in the network.

The algorithms proposed for LSP routing in MPLS networks are mostly state dependent ([3] $)$. In state dependent routing, the information about the status of the network is flooded through the network and routing tables are updated using this information. Event dependent routing algorithms, on the other hand, use the observed events to update their knowledge about the status of the network. Different event dependent routing schemes have been proposed and successfully used in TDM networks. Reference 4 presents an event dependent routing scheme for destination-based routing and shows the convergence with probability one of the proposed algorithm to the set of approximate Cesaro-Wardrop equilibria. An application of event dependent routing schemes in the MPLS networks has been presented in [5, 6. To the best of our knowledge, the performance of the routing scheme studied in [5, 6] can only be derived from discrete event simulation and there is no analytical approach for evaluating the performance of the algorithm. In reference [7, we present an alternative event dependent routing scheme with the application to explicit source routing in MPLS networks. The proposed algorithm is based on the Load Shared Sequential Routing (LSSR) where load sharing factors are updated using reinforcement learning techniques.

In this paper, we study the load share optimization problem both from user and system optimization perspectives in the LSSR model and give a computationally efficient method for solving these problems. The solution approach uses a recursion, governing the expected behavior of an $\epsilon$-optimal learning automata, to converge to the point where the Kuhn-Tucker conditions of the optimization problem are satisfied. The application of learning automata techniques in solving the load share optimization problem for the single class circuit-switched networks with fully connected topology and 2-hop alternate paths has been studied in 8 . The solution approach proposed in this paper can be used in the multi-rate traffic case with general network topology where some links may be shared among two or more alternate paths. Numerical results are presented comparing network blocking probabilities obtained from the user equilibrium and the system optimum load sharing factors in some example network topologies and traffic demands.

\section{Methodology}

This section briefly reviews the methodology and the algorithms that are used in this paper to compute the user equilibrium and the system optimum load sharing factors when LSSR is applied to route Label Switched Paths (LSPs) in a multi-rate MPLS network. 
Consider a learning automaton with $K$ actions and the following updating scheme 9]:

$$
p_{j}(t+1)= \begin{cases}p_{j}(t)+G\left[\delta_{j i}-p_{j}(t)\right] x(t) & j=1, . ., K-1 \\ 1-\sum_{i=1}^{K-1} p_{i}(t+1) & j=K\end{cases}
$$

where at time $t$, the $i$ th action is selected; $\delta_{j i}$, is Kronecher delta function and $x(t) \in[0,1]$ is the reward associated with the selected action. In the context of this paper, a reward is associated with a completed call attempt. In addition to being $\epsilon$-optimal [10], when the covariance between each pair $\left(p_{i}, p_{j}\right)\left(\sigma_{p_{i} p_{j}}^{2}=\right.$ $\left.E\left[p_{i} p_{j}\right]-E\left[p_{i}\right] E\left[p_{j}\right]\right)$ is negligible, this learning scheme has the following expected behavior:

$$
E\left[p_{j}(t+1)\right] \approx \begin{cases}E\left[p_{j}(t)\right]\left[1+G\left(s_{j}(t)-\sum_{k=1}^{K} s_{k}(t) E\left[p_{k}(t)\right]\right)\right] & j=1, \ldots, K-1 \\ 1-\sum_{j=1}^{K-1} E\left[p_{j}(t+1)\right] & j=K\end{cases}
$$

where,

$$
s_{i}(t)=E\left[x(t) \mid a(t)=a_{i}\right]
$$

This recursion governing the expected behavior will be used in solving optimization problems described in the next sections. This methodology has been previously employed to compute the user equilibrium and the system optimum routing solutions in datagram networks [1. It was shown that the recursion given in Equation (2), has fixed points which are in one-to-one correspondence with stable user equilibrium solutions, when the datagram network is modeled as in Gallager's classic paper [11. In references 9] and [10] the same recursion was derived to approximate the expected behavior of the action probabilities of the cross-correlation algorithm of Equation (1) in a stationary environment under slow learning conditions. The same cross-correlation learning algorithm and the recursion governing its expected behavior under slow learning conditions were applied to a variety of routing and flow control problems in data and ATM networks in a series of papers [12, 13 and 14. Recently Alanyali has provided an analysis of the behavior of distributed learning algorithms controlling a Markov process in [15].

The present paper differs from the results previously reported in that the network model used here is different, namely a general mesh topology supporting multi-rate LSPs. The multi-rate performance model of Greenberg and Srikant [16] is used where the LSPs are characterized by their Effective Bandwidth [17.

\section{$3 \quad$ RL-Based Load Shared Sequential Routing}

Load Shared Sequential Routing (LSSR) randomly partitions the class $s$ traffic load associated with origin 'o' and destination 'd' $\left(\lambda^{o, d, s}\right)$ into $n$ sub-streams using the set of load sharing factors (sub-stream selection probabilities), $\left\{\alpha_{1}^{o, d, s}, \ldots, \alpha_{k}^{o, d, s}\right\}$. Each sub-stream is then offered to a route tree which consists of one or more alternate paths. The alternate paths of each route tree are 


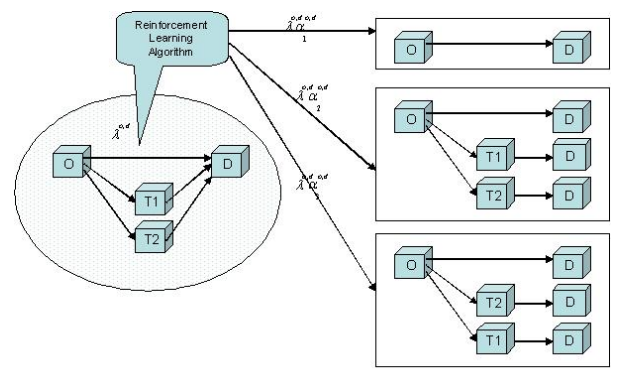

Fig. 1. LSSR Model

tried sequentially. If there is not enough bandwidth available on at least one link of one path, the request is forwarded to the next alternate path. This process is repeated until all alternate paths in the route tree have been tried sequentially. If all paths have been tried unsuccessfully, the request is lost and rejected from the network. A pictorial representation of the LSSR model is provided in Figure 1.

The LSSR model imposes no restriction on the load sharing factors other than non-negativity and

$$
\sum_{k} \alpha_{k}^{o, d, s}=1
$$

\subsection{Load Shared Sequential Routing, User Equilibrium Solution}

User equilibrium can be explained in terms of Wardrop equilibrium 18. In the LSSR context, let $\lambda^{o, d, s}$ represent the total class $s$ traffic load between origin ' $O$ ' and destination ' $d$ ' and let $L\left(\underline{\alpha}^{o, d, s}\right)$ be the cost of allocating the traffic $\lambda^{o, d, s}$ according to the load sharing factors $\underline{\alpha}^{o, d, s}$. The set of load sharing factors $\underline{\alpha}$ is at the Wardrop equilibrium if for each $\alpha_{i}^{o, d, s}, \alpha_{j}^{o, d, s}>0$, we have $L_{i}^{o, d, s}=L_{j}^{\overline{o, d, s}}$ and that if there exits a route tree with $\alpha_{\ell}^{o, d, s}=0$, then $L_{i}^{o, d, s} \leq L_{\ell}^{o, d, s}$. The load sharing factors $(\underline{\alpha})$ at the Wardrop Equilibrium are the solution to the following minimization problem:

$$
\min \overline{Z(\underline{\alpha})}=\sum_{o, d, s, k} \overline{L_{k}^{o, d, s}}
$$

$$
\begin{aligned}
\text { subject to } \quad \sum_{k=1}^{K_{o, d, s}} \alpha_{k}^{o, d, s} & =1 \\
\alpha_{k}^{o, d, s} \geq 0 & \left(v^{o, d, s}\right) \\
& \left(u_{k}^{o, d, s}\right),
\end{aligned}
$$

where $u_{k}^{o, d, s}$ and $v^{o, d, s}$ are Lagrangian multipliers and $\overline{L_{k}^{o, d, s}}$ is defined as:

$$
\overline{L_{k}^{o, d, s}}=\int_{0}^{\alpha_{k}^{o, d, s} \lambda^{o, d, s}} L_{k}^{o, d, s} d \alpha .
$$


From the Kuhn-Tucker conditions we have

$$
L_{k}^{o, d, s}=-\frac{v^{o, d, s}}{\lambda^{o, d, s}} \quad \forall \quad \alpha_{k}^{o, d, s}>0 .
$$

From this last condition, the user equilibrium solution is one that equalizes the cost on all the route trees on which traffic is offered $\left(\alpha_{k}^{o, d, s}>0\right)$.

\subsection{Load Shared Sequential Routing, System Optimization Problem Formulation}

In this case, the optimization problem has the following form:

$$
\begin{array}{rlr}
\min Z(\underline{\alpha}) & =\sum_{o, d, s, k} \lambda^{o, d, s} \alpha_{k}^{o, d, s} L_{k}^{o, d, s} & \\
\text { subject to } \sum_{k=1}^{K_{o, d, s}} \alpha_{k}^{o, d, s} & =1 & \left(v^{o, d, s}\right) \\
\alpha_{k}^{o, d, s} & \geq 0 & \left(u_{k}^{o, d, s}\right) .
\end{array}
$$

From the Kuhn-Tucker $(K K T)$ conditions we have

$$
\frac{\partial Z}{\partial \alpha_{k}^{o, d, s}}=-v^{o, d, s} \quad \forall \quad \alpha_{k}^{o, d, s}>0,
$$

which means that at the system optimum solution, for each $(o, d, s)$, the partial derivatives of network cost with respect to load sharing factors on all the route trees with $\alpha_{k}^{o, d, s}>0$ are equal.

\subsection{The Application of Learning Automata in Solving User and System Optimization Problems}

Assume there is a cross-correlation learning automata engine, $A^{o, d, s}$, associated with each $(o, d, s)$ with the set of actions of $A^{o, d, s}$ being the set of route trees available for routing bandwidth requests of class $s$ between pair $(o, d)$. Let $\alpha_{k}^{o, d, s}$ be the load sharing factor associated with $k$ th route tree of $(o, d, s)$.

As discussed in the previous section, from $K K T$ conditions of the user and system optimization problems, in the optimum solution, the partial derivatives of the cost function with respect to load sharing factors of those route trees with load sharing factor greater than zero are equal and less than or equal the partial derivative of the cost function with respect to load sharing factor of other route trees.

The recursion governing the expected behavior of cross-correlation learning automata is used for solving the optimization problems. First, the load sharing factors are arbitrarily initialized and traffic is distributed according to these load sharing factors over the set of route trees. The resulting blocking probabilities are calculated using the method of [16]. The load sharing factors are then updated using the recursion formula of Equation (2) with the following possible choices for $\underline{s}$ parameters: 
For user optimization problem :

$$
s_{k}^{o, d, s}(t)=1-\frac{L_{k}^{o, d, s}}{\sum_{r=1}^{K_{o, d, s}} L_{r}^{o, d, s}},
$$

For system optimization problem : $\quad s_{k}^{o, d, s}(t)=1-\frac{\frac{\partial Z}{\partial \alpha_{k}^{o, d, s}}}{\sum_{r=1}^{K_{o, d, s}} \frac{\partial Z}{\partial \alpha_{r}^{o, d, s}}}$.

The traffic distribution and resulting blocking probabilities are then updated and the process is repeated until $\left|\alpha^{\mathbf{o}, \mathbf{d}, \mathbf{s}}(t+1)-\alpha^{\mathbf{o}, \mathbf{d}, \mathbf{s}}(t)\right|$ is sufficiently small.

As seen from Equation (14), for the case of the system optimization problem, the calculation of $\underline{s}$ parameters implies the calculation of the partial derivatives of total blocking probability of the network with respect to each load sharing factor. The analytical formulation for these partial derivatives is complex and the numerical methods are subject to approximation errors which will affect the accuracy of the final results. To overcome this drawback, we consider a reformulation of the problem that leads to a more efficient approach for calculating $\underline{s}$ parameters.

\subsection{System Optimization Problem, Alternative Formulation}

In this formulation, we consider two sets of auxiliary variables: the average arrival rate for class $s$ on link $(i, j)\left(\mathbf{m}=\left[m_{i, j, s}\right]\right)$ and the class $s$ blocking probability of link $(i, j)\left(\mathbf{B}=\left[B_{i, j, s}\right]\right)$. The reformulated system optimization problem then becomes:

$$
\min _{\alpha, \mathbf{m}, \mathbf{B}} Z(\alpha, \mathbf{m}, \mathbf{B})=\sum_{o, d, s, k} \lambda^{o, d, s} \alpha_{k}^{o, d, s} L_{k}^{o, d, s}
$$

subject to

$$
\begin{array}{rlrl}
m_{i, j, s} & =\beta(\alpha, \mathbf{B}) & & \left(\eta_{i, j, s}\right) \\
B_{i, j, s} & =P b\left(m_{i, j, s}\right) & \left(\omega_{i, j, s}\right) \\
\sum_{k} \alpha_{k}^{o, d, s} & =1 & & \left(v^{o, d, s}\right) \\
\alpha_{k}^{o, d, s} & \geq 0 & & \left(u_{k}^{o, d, s}\right),
\end{array}
$$

where $\beta(\alpha, \mathbf{B})$ can be derived using the method presented in [16] and $\mathrm{Pb}(\mathbf{m})$ can be calculated using the Kaufman-Roberts recursion method.

The Kuhn-Tucker conditions are obtained by setting the derivative of Lagrangian of the problem $(H)$ with respect to $\alpha, \mathbf{m}$ and $\mathbf{B}$ equal to zero. This yields the following equations:

$$
\begin{aligned}
& \frac{\partial H}{\partial m_{i, j, s}}=0 \Rightarrow \eta_{i, j, s}=\sum_{r=1}^{S} \omega_{i, j, r} \frac{\partial P b\left(m_{i, j, r}\right)}{\partial m_{i, j, s}} \\
& \frac{\partial H}{\partial B_{i, j, s}}=0 \Rightarrow \omega_{i, j, s}=\sum_{p, q, s} \eta_{p, q, s} \frac{\partial \beta_{p, q, s}}{\partial B_{i, j, s}}-\sum_{o, d, k} \lambda^{o, d, s} \alpha_{k}^{o, d, s} \frac{\partial L_{k}^{o, d, s}}{\partial B_{i, j, s}}
\end{aligned}
$$




$$
\begin{gathered}
\frac{\partial H}{\partial \alpha_{k}^{o, d, s}}=0 \Rightarrow v^{o, d, s}=\sum_{(i, j) \in(o, d, s, k)} \eta_{i, j, s} \frac{\partial \beta_{i, j, s}}{\partial \alpha_{k}^{o, d, s}}-\lambda^{o, d, s} L_{k}^{o, d, s}-u_{k}^{o, d, s} \\
\alpha_{k}^{o, d, s} u_{k}^{o, d, s}=0 \quad \begin{array}{l}
\alpha_{k}^{o, d, s} \geq 0 \\
(i, j),(o, d) \in\{1, \ldots, N\}^{2}, s=1, \ldots, S, k=1, \ldots, K_{o, d, s}
\end{array}
\end{gathered}
$$

From these equations, for all route trees with $\alpha_{k}^{o, d, s}>0$, the terms $g_{k}^{o, d, s}=L_{k}^{o, d, s}-\sum_{(i, j) \in(o, d, s, k)} \eta_{i, j, s} \frac{1}{\lambda^{o, d, s}} \frac{\partial \beta_{i, j, s}}{\partial \alpha_{k}^{o, d, s}}$ are equal.

So if the values of $g_{k}^{o, d, s}$ can be calculated at each iteration of the recursion method discussed in the previous section, the load sharing factors can be updated using:

$$
s_{k}^{o, d, s}(t)=1-\frac{g_{k}^{o, d, s}}{\sum_{r=1}^{K_{o, d, s}} g_{r}^{o, d, s}} .
$$

To do so, the value of $\eta(i, j, s)$ need to be calculated. These values can be derived from the following compact set of equations:

$$
\left\{\begin{array}{l}
\underline{\eta}=\underline{\dot{\mathbf{P}} \mathbf{b} \omega} \\
\underline{\omega}=\underline{\dot{\beta} \eta}-\underline{\dot{\mathbf{L}} \lambda_{k}}
\end{array} \Rightarrow \underline{\eta}=\left(\underline{\dot{\beta}}^{-1}-\underline{\mathbf{P} \mathbf{b}}\right) \underline{\dot{\mathbf{L}}} \lambda_{k}\right.
$$

This set of equations models a hierarchical routing architecture where one centralized processor would be interconnected to the learning automata associated with every $(o, d, s)$. On a regular basis, the centralized processor collects the network information, updates the $\underline{\eta}$ parameters and distributes the updated parameters to the learning automata engines. Such a mechanism is capable of generating and maintaining a performance level equivalent to the one expected by the system solution. A pictorial representation of this architecture is given in Figure 2]

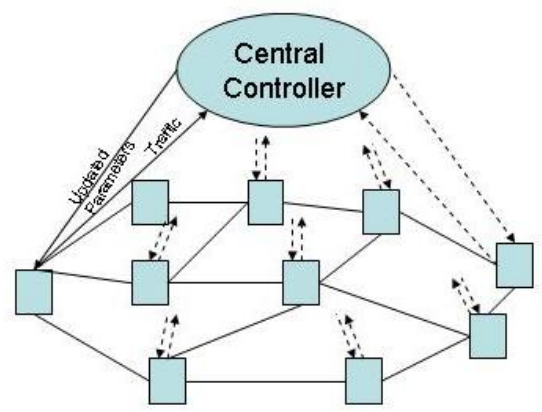

Fig. 2. Hierarchical Control Architecture 


\section{Numerical Results}

In this section, the performance of LSSR algorithm with user and system optimized load sharing factors are compared in an example 4-node network 2 different classes of service under full sharing assumption. Here, the capacity of each link is 150 trunks. The effective bandwidth for class 1 is equal to 1 trunk and for class 2 is equal to 2 trunks. For each $(o, d)$ pair, there are 5 sets of route-trees; one with only the direct path; two with the direct path and one of the alternate paths and two with the direct path and two other alternate paths. The order of the alternate paths is different in the last two route-trees. The stopping condition is when L1 norm of successive iterations differs by less than $10^{-5}$. The performance comparison of user and system optimized load sharing factors with normal traffic load (for each $(o, d, s), \lambda^{o, d, s}=41$ ) and heavy traffic load (for each $\left.(o, d, s), \lambda^{o, d, s}=50\right)$ with different $\underline{\eta}$ updating intervals are presented in Table (1D) and Table (2) repectively.

Table 1. 4-node 2-traffic class network under normal traffic

\begin{tabular}{|c|l|c|c|}
\hline $\begin{array}{c}\text { System } \\
\text { User }\end{array}$ & $\begin{array}{l}\mathrm{T} \text { Update } \\
\text { Interval }\end{array}$ & $\begin{array}{c}\text { Blk. } \\
\text { Prob. }\end{array}$ & $\begin{array}{c}\text { No. of } \\
\text { Iteration }\end{array}$ \\
\hline \hline Sys & $\mathrm{T}=1$ & .00152 & 12547 \\
Sys & $\mathrm{T}=20$ & .00155 & 14764 \\
Sys & $\mathrm{T}=200$ & .00154 & 14921 \\
Sys & $\mathrm{T}=1000000$ & .00161 & 16356 \\
User & & .00161 & 446 \\
\hline
\end{tabular}

Table 2. 4-node 2-traffic class network under heavy traffic

\begin{tabular}{|c|l|c|c|}
\hline $\begin{array}{c}\text { System/T Update } \\
\text { User }\end{array}$ & $\begin{array}{l}\text { Blk. } \\
\text { Interval }\end{array}$ & $\begin{array}{c}\text { No. of } \\
\text { Prob. }\end{array}$ & Iteration \\
\hline \hline Sys & $\mathrm{T}=1$ & .0727 & 2582 \\
Sys & $\mathrm{T}=20$ & .0741 & 2150 \\
Sys & $\mathrm{T}=200$ & .0784 & 3563 \\
Sys & $\mathrm{T}=1000000$ & .0912 & 5327 \\
User & & .1232 & 737 \\
\hline
\end{tabular}

In the next set of experiments, a 9-node network topology is considered with fully isolated maximum allocation bandwidth constraint model. As different classes are fully isolated, we consider only one of the classes of service. For each $(o, d)$ pair, sets of route-trees compose of the direct path and one or two alternate paths. The stopping condition is when the L1 norm of the load sharing factors in successive iterations differs by less than $10^{-5}$. Here again, for the system optimization problem, the parameter $\eta$ is updated once every $T$ iterations. The blocking probability results for 3 different updating intervals with normal and heavy traffic loads are summarized in Table 3 and Table 4

As seen from the presented results, for the case of normal traffic load, the user equilibrium and the system optimum solutions give similar network performance while in the case of heavy traffic load, the system optimum solution gives better performance in terms of blocking probability. Moreover, the interval between updating $\underline{\eta}$ parameters has a negligible impact on the final blocking probability of the network. This in turn means that the system optimal solution can be obtained using a few number of updating $\underline{\eta}$ parameters. One should note that for the system optimum solution, the computational cost depends on the number of iterations and the computation cost of deriving the $\underline{\eta}$ parameters. 
Table 3. 9-Node Network Nominal Rate

\begin{tabular}{|c|l|c|c|}
\hline $\begin{array}{c}\text { System/ } \\
\text { User }\end{array}$ & $\begin{array}{l}\mathrm{T} \text { Update } \\
\text { Interval }\end{array}$ & $\begin{array}{c}\text { Blk. } \\
\text { Prob. }\end{array}$ & $\begin{array}{c}\text { No. of } \\
\text { Iteration }\end{array}$ \\
\hline Sys & $\mathrm{T}=10$ & .00448 & 17363 \\
Sys & $\mathrm{T}=100$ & .00448 & 19483 \\
Sys & $\mathrm{T}=1000000$ & .00451 & 21436 \\
User & & .00452 & 1849 \\
\hline
\end{tabular}

Table 4. 9-Node Network Heavy Traffic

\begin{tabular}{|c|l|c|c|}
\hline $\begin{array}{c}\text { System } \\
\text { User }\end{array}$ & $\begin{array}{l}\mathrm{T} \text { Update } \\
\text { Interval }\end{array}$ & $\begin{array}{c}\text { Blk. } \\
\text { Prob. }\end{array}$ & $\begin{array}{c}\text { No. of } \\
\text { Iteration }\end{array}$ \\
\hline Sys & $\mathrm{T}=10$ & .01868 & 8679 \\
Sys & $\mathrm{T}=100$ & .01868 & 10376 \\
Sys & $\mathrm{T}=1000000$ & .01875 & 12543 \\
User & & .01879 & 2385 \\
\hline
\end{tabular}

\section{Summary and Conclusion}

In this paper, an event dependent routing method based on load shared sequential routing for MPLS networks was presented and the problem of optimizing the load sharing factors with the objective of minimizing the blocking probability either in full sharing case or the case where MAM is used as the bandwidth constraint model was discussed. A new method for solving the optimization problem both from the user and the system optimization perspectives was given.

In general, the user equilibrium and the system optimum solutions yield different routing solutions. Since the user solution is derived solely from local information, the resulting network blocking probability will generally be higher than that of the system optimum solution. However, in some cases, such as the cases studied in this report for the networks operating in nominal traffic loads, the difference can be relatively small.

While the system solution can only be derived with the global information, this paper has shown that it is possible to decompose the centralized optimization process into relatively smaller sub-processes. The centralized operations are restricted to the evaluation of the shadow prices. All other operations can be performed through decentralized sub-processes.

\section{References}

[1] Mason, L.G.: Equilibrium flows, routing patterns and algorithms for store-andforward networks. Journal of Large Scale systems 8 (1985) 187-209

[2] LeFaucheur, F., Lai, W.: Requirements for Support of Differentiated Servicesaware MPLS Traffic Engineering. RFC 3564 (2003)

[3] Marzo, J.L., Calle, E., Scoglio, C., Anjah, T.: QoS online routing and MPLS multilevel protection: a survey. IEEE Communications Magazine (2003) 126-132

[4] Borkar, V., Kumar, P.: Dynamic Cesaro-Wardrop equilibration in networks. IEEE Trans. on Automatic Control (2003) 382-396

[5] Ash, G.R.: Performance evaluation of QoS-routing methods for IP-based multiservice networks. Computer Communications 26 (2003) 817-833

[6] Ash, G.R.: Traffic Engineering and QoS Optimization of Integrated Voice \& Data Networks. Morgan Kaufmann Publishers Inc., San Francisco, CA, USA (2006)

[7] Heidari, F., Mannor, S., Mason, L.G.: Reinforcement learning-based load shared sequential routing. IFIP-Networking, to appear (2007)

[8] Brunet, G.: Optimisation de l'acheminement séquentiel non hiérarchique par automates intelligents. Master's thesis, INRS-Telecommunication (1991) 
[9] Mason, L.G.: An optimal learning algorithm for S-model environments. IEEE Trans. on Automatic Control 18 (1973) 493-496

[10] Mason, L.G.: An optimal learning algorithm employing cross-correlation. In: Conference Record, Joint Automatic Control Conference. (1973) 572-578

[11] Gallager, R.: A minimum delay routing algorithm using distributed computation. Communications, IEEE Trans. on 25 (1977) 73-85

[12] Cotton, M., Mason, L.G.: Adaptive isarithmic flow control in fast packet switching networks. IEEE Trans. on Communications 43 (1995) 1580-1590

[13] Pelletier, A., Cotton, M., Mason, L.G.: Combined adaptive routing and flow control in fast packet switching networks. In: Proceedings of COMCON 4, Rhodes, Greece. (1993) 557-569

[14] Vazquez-Abad, F.J., Mason, L.G.: Decentralized adaptive flow control of highspeed connectionless data networks. Journal of Operations Research 47 (1999) 928-942

[15] Alanyali, M.: Learning automata in games with memory with application to circuit-switched routing. In: Decision and Control, 2004. CDC. 43rd IEEE Conference on. Volume 5. (2004) 4850- 4855

[16] Greenberg, A.G., Srikant, R.: Computational techniques for accurate performance evaluation of multirate, multihop communication networks. IEEE/ACM Trans. on Networking 5 (1997) 266-277

[17] Kelly, F.P.: Effective bandwidths at multi-class queues. Queueing Systems: Theory and Applications 9 (1991) 5-15

[18] Wardrop, J.G.: Some theoretical aspects of road traffic research. In: Proceedings of the Institution of Civil Engineers, Part II. (1952) 325-378 